\title{
Center of pressure limb path differences for the detection of lameness in dogs: a preliminary study
}

Sergio López ${ }^{1}$, José M. Vilar ${ }^{1,4^{*}}$ (D) Mónica Rubio ${ }^{2}$, Joaquin J. Sopena ${ }^{2}$, Elena Damiá2 ${ }^{2}$ Déborah Chicharro², Angelo Santana ${ }^{3}$ and José M. Carrillo ${ }^{2}$

\begin{abstract}
Background: The limb center of pressure (COP) path measures and quantifies the load distribution within a limb in a still or moving subject. Under this premise, the aim of this study was to test whether data derived from this parameter could detect the differences between sound and lame limbs in unilaterally lame dogs with elbow dysplasia.

To accomplish this purpose, ten unilaterally lame dogs of similar conformation were walked over a pressure platform. Next, the COP path, in relation to the position of sound and lame limbs, was measured in a coordinate system over a standard paw template obtained by pedobarography during the whole support phase. To compare variables, force platform data (peak vertical force and vertical impulse) from the same animals were obtained. Sound and lame limb statokinesiograms were also obtained while the animals stood still.
\end{abstract}

Results: The statistical analysis clearly showed that COP in lame limbs start cranially and were shorter than sound limbs. In addition, the value of the COP excursion index was lower in lame limbs. Finally, the area of statokinesiograms was greater in lame limbs.

Conclusion: This methodology based in limb COP characteristics serves to discriminate between sound and lame limbs in dogs with elbow dysplasia.

Keywords: Balance, Center of pressure, COP, Dog, Statokinesiogram

\section{Background}

Various methods to analyze the locomotor status within the veterinary field have been developed in order to generate useful parameters from both kinematic and/ or kinetic perspectives. These methodologies should be able to provide accurate and reliable data and, if possible, form a set of parameters that will allow for the normal/abnormal static/dynamic events from a wide perspective. This invariably requires the use of more sophisticated systems [1].

These data should ultimately serve to detect lameness, and, among them, the center of pressure (COP) position

\footnotetext{
* Correspondence: jose.vilar@ulpgc.es

${ }^{1}$ Instituto Universitario de Investigaciones Biomédicas y Sanitarias,

Universidad de las Palmas de Gran Canaria, Arucas, Las Palmas, Spain

${ }^{4}$ Departamento de Patología Animal, Universidad de las Palmas de Gran

Canaria, Arucas, Las Palmas, Spain

Full list of author information is available at the end of the article
}

may be considered the net output variable of interaction between all of the forces and torques that occur in the body (bCOP) or limb (lCOP) and its inertial properties. The COP position over time is named the COP path. This parameter quantifies the dynamic load distribution under the foot [2]. The ICOP path characteristics obtained in moving subjects provide insights into foot dynamics during the support phase of gait in human and, potentially, in animal species [3-6]. In this sense, it has been able to reliably detect biomechanical modifications due to neurological deficits, such as Parkinson's [7], Hemiparesis [8] or even pain [3], in humans.

The main ICOP pathway characteristics that have been reported as useful are: 1) craniocaudal COP excursion (measured as an initial and final COP relative coordinates) [8]; 2) lateromedial displacement of the ICOP by means of the center of pressure excursion index (CPEI),

(c) The Author(s). 2019 Open Access This article is distributed under the terms of the Creative Commons Attribution 4.0 International License (http://creativecommons.org/licenses/by/4.0/), which permits unrestricted use, distribution, and reproduction in any medium, provided you give appropriate credit to the original author(s) and the source, provide a link to the Creative Commons license, and indicate if changes were made. The Creative Commons Public Domain Dedication waiver (http://creativecommons.org/publicdomain/zero/1.0/) applies to the data made available in this article, unless otherwise stated. 
which represents the ICOP path lateromedial excursion relative to limb width and multiplied by 100 to obtain this data in terms of percentage $[3,9]$.

The COP path can be also obtained in a standing position and records its resultant area during a determinate period of time. This parameter is named statokinesiogram, and its value shows body or limb balance [10].

In the veterinary field, previously published studies only examine the bCOP path [11-13]; more recently, the bCOP path's efficacy for the detection of lameness in ponies at walk has been settled [14]. In dogs, bCOP modifications in unilaterally lame animals with elbow dysplasia (ED) have also been reported [15].

Regarding ED, this is a complex syndrome, where different factors could lead to a growth incongruence between the radius and ulna. Over time, ED causes joint damage, pain, and lameness [16, 17].

The hypothesis of this study was to prove that certain ICOP path characteristics are different in lame and sound limbs in dogs at walk and while standing still. For this reason, the aim of this study was to set a number of ICOP pathways -derived data that could serve to detect lameness in dogs with unilateral ED.

\section{Methods}

\section{Animals}

This study utilized 10 client-owned, adult dogs with similar conformation (2 rottweiler, 3 labrador retriever, 1 golden retriever, 2 german shepherd, 2 belgian shepherd). The body weight of the enrolled dogs ranged from 30 to $41,8 \mathrm{~kg}$, and the ages were from 3 to 9 years.

Inclusion criteria comprised of the presence of weight-bearing unilateral forelimb lameness due to OA secondary to elbow dysplasia. The lameness of every dogs reached a score of 3-4 in a scale of $0-5$ [18].

Furthermore, no medication could have been administered 1 month prior to the analysis.

To confirm or rule out OA, three standard radiographic views of both elbow joints (a lateral extension, lateral flexion, and a $15^{\circ}$ oblique craniomedial caudolateral) [19] were taken under sedation with dexmedetomidine $10 \pm$ $20 \mu \mathrm{g} / \mathrm{kg}$ (Dexdomitor, zoetis, Spain). Standard radiographs of stifle and hip joints were also taken in order to exclude other reasons for the observed clinical signs.

A complete clinical evaluation (physical examination, including vital signs and neurologic and orthopedic exams) assured that general health was otherwise normal.

\section{Pressure platform study}

A Pressure platform (EPS/R1, Loran Engineering, Bologne, Italy) was used for this study. This device contains a total of 2096 pressure sensors of $1 \mathrm{~cm} 2$ distributed in an area of $48 \times 48 \mathrm{~cm}$. The range of pressure was set from 30 to $400 \mathrm{kPa}$.

The procedure for the dynamic and static pressure platform analysis has been previously published $[15,20]$; briefly, dogs were leash guided by their owners over the pressure platform at a walk (velocity $1.2 \pm 0.2 \mathrm{~m} / \mathrm{s}$; acceleration $\pm 0.2 \mathrm{~m} / \mathrm{s}^{2}$ ). Velocity and acceleration were measured with a motion sensor (PS-2103A, Pasco ${ }^{\circ}$, California, USA) placed within the dogs trajectory. Three trials were recorded at a sampling frequency of $100 \mathrm{~Hz}$ from each dog. A trial was considered valid when the studied limb fully supported over the pressure platform and when the dog walked next to the owner without pulling on the leash and without head turns. The pressure platform was interfaced with a dedicated computer using Biomech ${ }^{\bullet}$ (Loran Engineering, Bologna, Italy) software. Once the images were isolated, the paws' length was normalized to a fixed value of $9 \mathrm{~cm}$, and width was then proportionally modified. Measurements were taken with a reference to an X-Y coordinate system.

Statokinesiograms were obtained while the dogs were placed in a quiet stance with their thoracic limbs over the pressure platform, perpendicular to the ground. The dog's owner remained in front of the animal to attract the dog's attention at a close distance. Three trials of 20-s recordings were obtained from each animal. A trial was considered valid when the animal remained with immobile limbs, tail and head during the whole $20 \mathrm{~s}$ recording procedure.

The following were the obtained measurements (Fig. 1):

1. Caudal margin $(\mathrm{Cm})$ : defined as the distance between the most caudal limit of the paw print and the most caudal limit of the ICOP path.

2. ICOP pathway length (e): the length of the line that joins the recorded points of the lCOP trajectory. Measured in $\mathrm{cm}$.

3. Craniocaudal index $(\mathrm{CrCI})$ : determines the $\mathrm{COP}$ length (b) related to the paw length (a). This is obtained with the following formula: $\%=(b / a) \times$ 100. Expressed as a percentage.

4. Center of the pressure excursion index (CPEI): determines the lateromedial excursion of the COP (c) related to the paw width (d). The formula was the following: $\%=(\mathrm{c} / \mathrm{d}) \times 100$. Expressed as a percentage.

Higher values of all the above parameters are associated with better limb support $[3,8,9]$.

5. statokinesiograms: defined as the area determined by an ellipse that contains $90 \%$ of the recorded points of the COP trajectory [10]. Measured in $\mathrm{mm} 2$, a lower value means more stability $[15,21]$. 


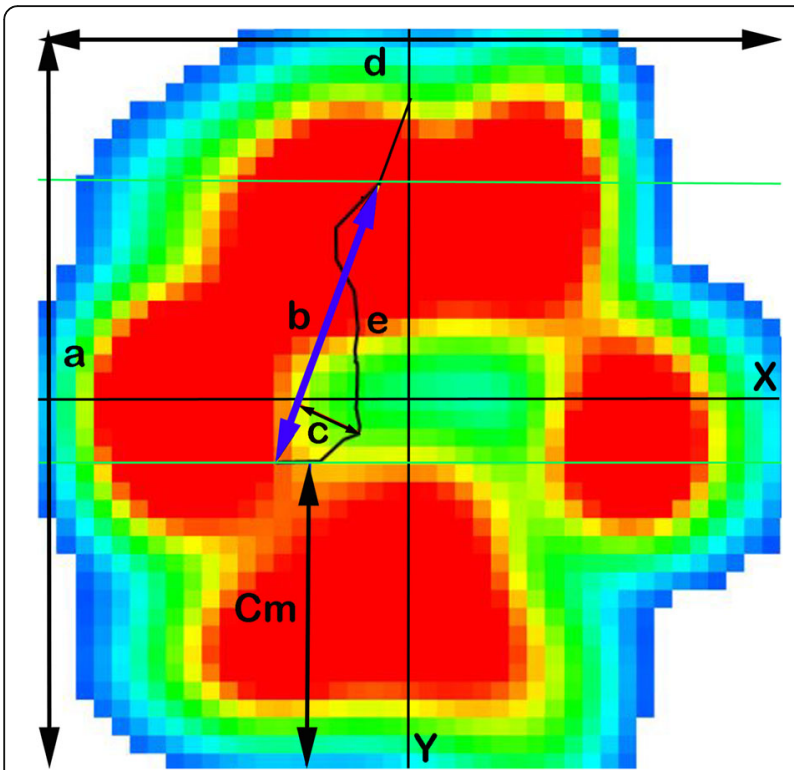

Fig. 1 Paw podobarographic print with coordinate system and measurements made. X: X coordinate; Y: Coordinate; a: paw length; b: COP length; c: ICOP width; d: paw width; e: COP path length; Cm: caudal margin

\section{Force platform analysis}

A force platform (Pasco, California, USA) was placed adjacent to the pressure platform in such a way that recordings from animals were performed in the same session. DataStudio software (Pasco, California, USA) was used to obtain PVF $(\mathrm{N})$ values from three valid trials. Mean values were normalized to body weight (\%BW).

\section{Statistical analysis}

For the data analysis, a linear mixed effects model was considered: for each response variable (COP Length, CPEI, etc), the status of the limb (lame/sound) is a fixed effects factor, while the dog is a random effects factor.

The model is as follows:

$$
\begin{aligned}
& \begin{aligned}
y \_i j k & =\mu \_i+b_{-} j+\varepsilon_{-} i j k, i=1, \ldots, 2 j=1, \ldots, 10, k \\
& =1, \ldots, 3
\end{aligned} \\
& \text { b_i } \approx N\left(0, \sigma \_b\right) \quad \varepsilon_{-} i j k \approx N(0, \sigma)
\end{aligned}
$$

where:

1. $\mathrm{y}_{\text {_ijk }}$ is the $\mathrm{k}$-th measure $(\mathrm{k}=1,2,3)$ on the limb $\mathrm{i}$ ( $\mathrm{i}$ = sound/lame) of the $\operatorname{dog} j(j=1 . .10)$

2. $\quad \mu$ i i is the (fixed) effect of limb status $i$. This parameter represents the mean value of the variable in the sound (lame) limb.

3. $b \_j$ is the (random) effect of dog $j$. Values of $b \_j$ are supposed to be normally distributed with mean 0 and standard deviation $\sigma \_b$, so $\sigma \_b$ is the variability in the response of the dogs.

4. $\varepsilon$ _ijk is the residual in the measure ijk. This variable is assumed to be normally distributed with the mean 0 and standard deviation $\sigma$.

Statistical analysis was performed with ' $R$ ' statistical language and environment, version 3.3.2. (https://www. R-project.org/). For assessing the validity of the model, a Shapiro-Wilk test is applied to test the normality of the residuals, and a Levene test is used to test homoscedasticity.

\section{Results}

Mean weight ( \pm SD) was $37.08 \pm 3.76 \mathrm{~kg}$, and age was $5.80 \pm 1.99$ years. The mean $( \pm$ SD) values and $95 \% \mathrm{CI}$ of all obtained parameters are shown in Table 1. All data were normally distributed and homoscedastic $(p \geq 0.25$ and $p \geq 0.12$, respectively).

Significant differences between LL and CL were found in all cases $(<0.0001)$; concretely, a higher value of $\mathrm{Cm}$ and a lower COP Length, COP Path Length, and $\mathrm{CrCI}$ values in LL were observed when compared with CL. In the same manner, CPEI in LL were also lower than CL (Fig. 2, Additional file 1).

In agreement with the data shown above, PVF and VI values also showed significant differences between LL and CL $(p \leq 0.0001)$ (Table 1). PVF and VI data were also normally distributed and homoscedastic $(p \geq 0.64$ and $p \geq 0.51$, respectively).

Table 1 Mean \pm SD, 95\% confidence interval and difference between LL and CLs for CM, Cop Path Length, CrCl, PVF, VI and statokinesiograms. ${ }^{a}$ means significant difference

\begin{tabular}{llll}
\hline & $\mathrm{LL}$ & $\mathrm{CL}$ & Difference \\
\hline Cm (\%) & $44.85 \pm 5.12$ & $31.13 \pm 7.61$ & ${ }^{\mathrm{a}} 13.72 \pm 0.94$ \\
& $40.86,48.84$ & $27.14,35.12$ & $11.83,15.61$ \\
COP Path Length (\%) & $42.00 \pm 4.94$ & $55.68 \pm 9.92$ & ${ }^{\mathrm{a}} 13.69 \pm 0.97$ \\
& $37.05,46.95$ & $50.73,60.63$ & $11.74,15.63$ \\
CrCl (\%) & $31.07 \pm 4.49$ & $44.01 \pm 6.75$ & ${ }^{a} 12.94 \pm 1.23$ \\
& $28.08,34.06$ & $41.02,47.01$ & $10.47,15.42$ \\
CPEI (\%) & $4.57 \pm 1.65$ & $9.30 \pm 1.78$ & ${ }^{a} 4.73 \pm 0.35$ \\
& $3.65,5.49$ & $8.38,10.22$ & $4.02,5.44$ \\
PVF (\%) & $32.72 \pm 4.66$ & $71.12 \pm 3.57$ & ${ }^{a} 38.40 \pm 0.78$ \\
& $31.13,34.31$ & $69.53,72.71$ & $36.84,39.96$ \\
VI (\%) & $13.49 \pm 1.32$ & $22.93 \pm 1.58$ & ${ }^{a} 9.44 \pm 0.24$ \\
& $12.80,14.18$ & $22.24,13.62$ & $8.96,9.92$ \\
Statokinesiogram (mm $\left.{ }^{2}\right)$ & $16.18 \pm 6.10$ & $5.70 \pm 3.43$ & ${ }^{a} 10.48 \pm 0.75$ \\
& $13.16,19.19$ & $2.68,8.71$ & $8.98,11.98$ \\
\hline
\end{tabular}

$\mathrm{Cm}$ Caudal margin, $\mathrm{CrCl}$ Craniocaudal index, $\mathrm{CPEl}$ Center of pressure excursion index 


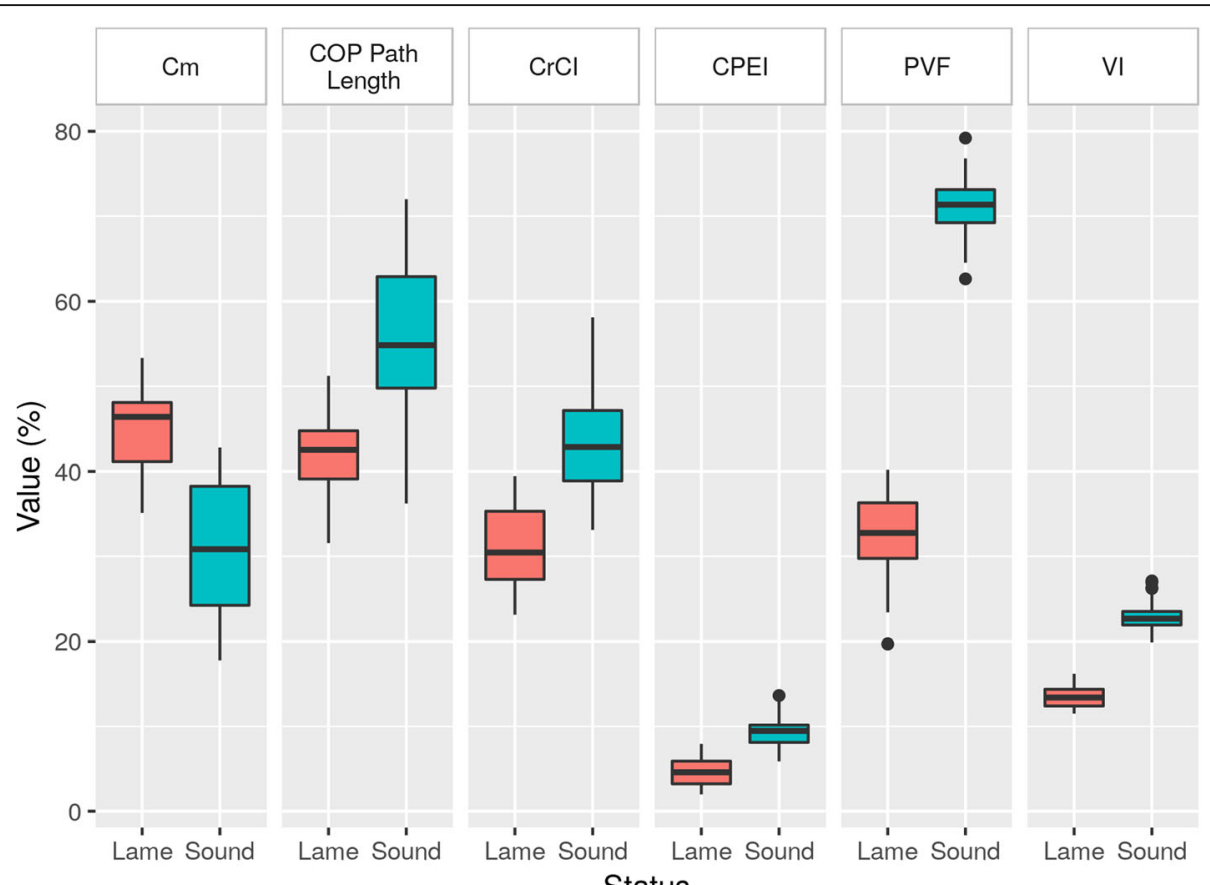

Status

Fig. 2 Boxplots showing differences in dynamic parameters between $L L$ and $C L$. As can be seen, $C m$ values are lower in $C L$, while $C O P$ path Length, $\mathrm{CrCl}$ and $\mathrm{CPEl}$ indexes are higher when compared with LL. This also occurs in PVF and VI values

Finally, the area from the statokinesiograms showed a higher value in LL (Fig. 3, Additional file 2). Additionally, a craniomedial COP slope was observed in both LL and CL when COP length was measured (Fig. 1, blue arrow).

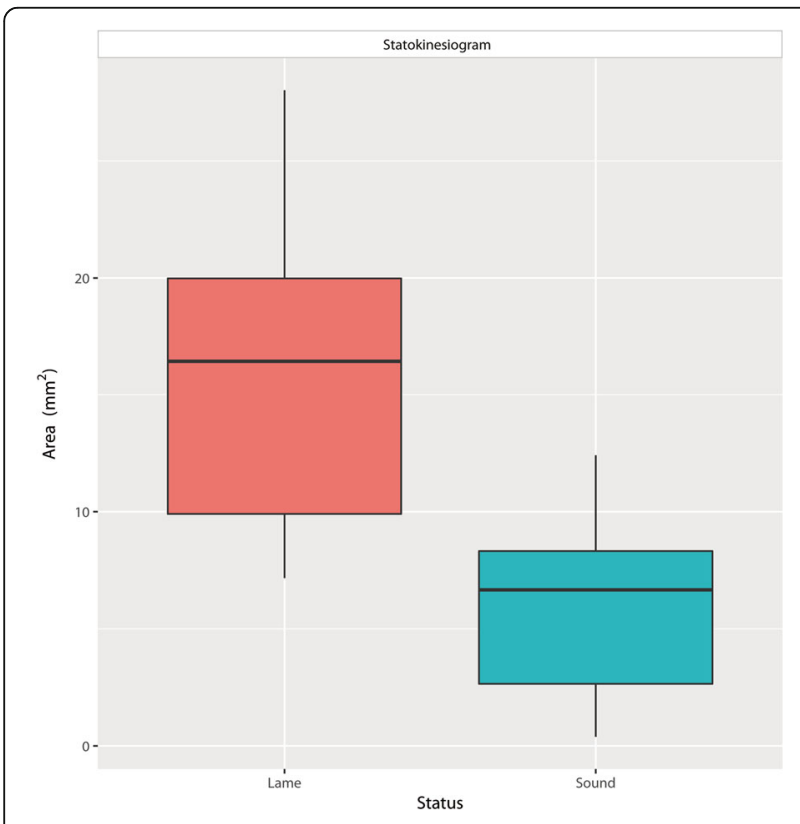

Fig. 3 Boxplots of statokinesiogram (static) values of $L L$ and $C L$. Area of $L L$ is higher than $L L$ i.e., more instable

\section{Discussion}

Our results provide a novel insight into the adaptive changes in ICOP characteristics in unilaterally lame dogs with ED.

To the best of our knowledge, no other previous studies exist regarding the clinical implications of dynamic and static ICOP path characteristics in lame dogs.

Limb weight load amount could be influenced by the gait speed or cadence and, consequently, could alter COP path patterns [22]. Acknowledging this possibility, we performed the study in a narrow range of velocity and acceleration and tried to enroll similarly sized animals in order to minimize severe cadence discrepancies.

Once the data were obtained, we assumed that measurements on caudocranial and mediolateral COP displacement would provide four basic differences between LL and CL regarding:

1) The extent of net forward lCOP path progression. Based in our results, ICOP path in LL is shortened and cranialized compared with CL. This is in concordance with other authors' findings [8]. As made evident by the data, a larger $\mathrm{Cm}$ directly implies a shorter COP path length. This is invariably due to a shortened swing phase by a lack of limb extension, meaning the limb lands more vertically at the start of the braking phase [23]. This event prevents the metacarpal pad to exert a correct load absorption, expanding with the 
increase of weight-bearing when the limb lands [24, 25]. The impact shock could be, in the last instance, potentially transferred to muscles higher up the limb [5].

2) Net mediolateral lCOP deviation. As reported in previous research [26], a higher CPEI in CL is determined by an increased pad deformation, given that pad expansion is a direct response to weight loading. This effect has also been observed in human feet [9] and equine hooves [27].

3) Statokinesiograms. A greater area determines more instability [15]. This finding, although previously in reference to the body, remains true for limbs as well, since the area was greater in LL.

4) The lCOP direction of progression in both sound and lame limbs. As stated above, ICOP path described a certain angle (slope) as it pursued craniomedially with respect to the longitudinal axe of the paw. A possible explanation for this finding may be that the ICOP path follows the direction of the body's center of mass and not the craniocaudal paw axe, which corresponds to other reports in humans [28].

Another interesting finding was that the ICOP caudocranial displacement is constant during the support phase, but velocity is not (Additional file 1), which coincides with reports in human research regarding sound limbs [8]. In the present study, this characteristic was evident not only in CL but also in LL.

In humans, longitudinal COP displacement corresponds to $83 \%$ of foot length and $18 \%$ of foot width [28]; their equivalent values in $\mathrm{CL}$ of our study with dogs were about 44\% (CrCI) and 9\% (CPEI), respectively, which is approximately half. Two facets could explain these differences: 1 - that humans have plantigrad support, which starts in the calcaneus bone, whereas in dogs the support is digitigrade; 2- human bipedalism determines full load transfer to the support limb when walking, whereas dogs walk with two (or even three) limbs simultaneously sharing the load support.

The following are some limitations in our study:

1. The ICOP path patterns in sound limbs cannot be extrapolated to limbs from sound dogs. As in lame dogs, sound limb patterns are showing compensatory movements. For the same reason, data from unilaterally lame limbs should not be extrapolated to bilateral lameness.

2. Compensatory weight redistribution in lame dogs not only implies to the contralateral limb, as has been well established in dogs and horses [29, 30]; thus, it would be useful to obtain hind limb ICOP path values in a subsequent study. Moreover, it should be determined if any correlations exist between the ICOP path values with the lameness degree or lameness origin. Unfortunately, the relatively large dog sizes impede the simultaneous analysis of more than two limbs, and a larger platform pressure mat would be essential.

3. Parameters, such as Cm and CPEI, need to be qualitative and not quantitatively considered, given that cut-points were not defined in our study, although significant differences were found in our study between CL and LL. To establish an accurate limit value for soundness or lameness, a higher number of patients with the same characteristics (weight, conformation, or even breed) are necessary, as reported by others authors in similar human studies [4].

4. Finally, the number of ICOP characteristics assessed could represent a "signature" diagnosis of ED, where the kinetic parameters to detect it have been previously proven [23]. This also means that COP patterns in other musculoskeletal and neurodegenerative disorders could be quite different, which needs further investigation.

\section{Conclusion}

This study showed that the ICOP path in LL is shorter, cranialized, and with smaller mediolateral excursion when compared with SL in dogs with unilateral ED. In addition, the ICOP path follows a craniomedial direction and not the paw longitudinal axe in both LL and CL. Its progression velocity is not constant.

\section{Additional files}

Additional file 1: Video S1. Limb and body statokinesiograms from a dog with a left limb lameness. As can be seen, the area of ellipse (18.28 $\mathrm{mm}^{2} \mathrm{Vs} 8,33 \mathrm{~mm}^{2}$ ) in the left (red) $\mathrm{LL}$ is greater than the right (blue) $\mathrm{CL}$. In the center (green) the body statokinesiogram can also be seen. (MP4 $3152 \mathrm{~kb}$ )

Additional file 2: Video S2. Simultaneous videosequence of support phase in a $C L$ (left) and LL (right). The ICOP (black point) path in LL starts more cranially and therefore shortened. (MP4 $650 \mathrm{~kb}$ )

\section{Abbreviations \\ bCOP: Body Center of Pressure; CL: Sound limb; Cm: Caudal margin; COP: Center of pressure; CPEl: Center of pressure excursion index; $\mathrm{CrCl}$ : Craniocaudal index; ED: Elbow dysplasia; ICOP: Limb Center of Pressure.; LL: Lame limb; PVF: Peak vertical force; VI: Vertical impulse}

\section{Acknowledgments}

The authors thank Amanda Hand for translation and editing. We would also like to thank the dog's owners for their collaboration. Thanks also to the Cátedra García Cugat for its technical support. 


\section{Availability of data and materials}

All data supporting our findings are included in the manuscript. If readers need additional information and/or data sets, they will be provided by the corresponding author upon reasonable request.

\section{Authors' contributions}

$J M V$, JS, and JMC conceived and designed the experiments; MR, ED and DC performed the clinical and radiological analyses; JMV and SL performed the pressure platform analysis; AS analyzed the data; all authors read and approved the final manuscript.

\section{Authors' information}

Not applicable

\section{Ethics approval and consent to participate}

The research protocol was revised and authorized by the Ethical Committee of Animal Welfare at the Instituto Universitario de Investigaciones Biomédicas y Sanitarias of the Universidad de Las Palmas de Gran Canaria (IUIBS 14/2017) in compliance with the Directive 2010/63/EU of the European Union. Dog owners were informed of the study and signed a consent to participate in the study, including all performed procedures.

\section{Consent for publication}

Not applicable

\section{Competing interests}

The authors declare that they have no competing interests.

\section{Publisher's Note}

Springer Nature remains neutral with regard to jurisdictional claims in published maps and institutional affiliations.

\section{Author details}

'Instituto Universitario de Investigaciones Biomédicas y Sanitarias, Universidad de las Palmas de Gran Canaria, Arucas, Las Palmas, Spain. 2Departamento Medicina y Cirugía Animal, Cátedra García Cugat, Universidad Cardenal Herrera-CEU, CEU Universities, Valencia, Spain. ${ }^{3}$ Departamento de Matemáticas, Universidad de las Palmas de Gran Canaria, Las Palmas, Spain. ${ }^{4}$ Departamento de Patología Animal, Universidad de las Palmas de Gran Canaria, Arucas, Las Palmas, Spain.

Received: 18 July 2018 Accepted: 24 April 2019

Published online: 08 May 2019

\section{References}

1. Kotti M, Duffell LD, Faisal AA, McGregor AH. Detecting knee osteoarthritis and its discriminating parameters using random forests. Med Eng Phys. 2017:43:19-29

2. Alexander IJ, Campell KR. Dynamic assessment of foot mechanics as an adjunct to orthotic prescription. In: Wolf SL, editor. The biomechanics of the foot and ankle. 1st ed. Philadelphia: FA Davis; 1990. p. 148-52.

3. Riskowski JL, Dufour AB, Hagedorn TJ, Hillstrom HJ, Casey VA, Hannan MT. Associations of foot posture and function to lower extremity pain: results from a population-based foot study. Arthritis Care Res. 2013;65:1804-12.

4. Grundy M, Tosh PA, Mcleish RD, Smidt L. An investigation of the centers of pressure under the foot while walking. J Bone Joint Surg (B). 1975;57:98-103.

5. Nauwelaerts S, Hobbs SJ, Back W. A horse's locomotor signature: COP path determined by the individual limb. PLoS One. 2017;12(2):e0167477.

6. LeBlanc C, Tobalske B, Szkotnicki B. Harlander-Matauschek a. locomotor behavior of chickens anticipating incline walking. Front Vet Sci. 2017;4:233.

7. Kim DY, Hwang SH, Kim MG, Song JH, Lee SW, Kim IK. Development of Parkinson patient generated data collection platform using FHIR and loT devices. Stud Health Technol Inform. 2017;245:141-5

8. Robain G, Valentini F, Renard-Deniel S, Chennevelle JM, Piera JB. A baropodometric parameter to analyze the gait of hemiparetic patients: the path of center of pressure. Ann Readapt Med Phys. 2006:49:609-13.

9. Yoon HK, Park KB, Roh JY, Park HW, Chi HJ, Kim HW. Extraarticular subtalar arthrodesis for pes planovalgus: an interim result of 50 feet in patients with spastic diplegia. Clin Orthop Surg. 2010;2:13-21.
10. Scoppa F, Capra R, Gallamini M, Shiffer R. Clinical stabilometry standardization: basic definitions--acquisition interval--sampling frequency. Gait Posture. 2013;37:290-2.

11. Nauwelaerts S, Malone SR, Clayton HM. Development of postural balance in foals. Vet J. 2013;198(Suppl 1):e70-4.

12. Clayton HM, Nauwelaerts S. Effect of blindfolding on Centre of pressure variables in healthy horses during quiet standing. Vet J. 2014;199:365-9.

13. Clayton HM, Buchholz R, Nauwelaerts S. Relationship between morphological and stabilographic variables in standing horses. Vet J. 2013; 198(Suppl 1):e65-9.

14. Pitti L, Oosterlinck M, Diaz-Bertrana ML, Carrillo JM, Rubio M, Sopena J, Santana A, Vilar JM. Assessment of static posturography and pedobarography for the detection of unilateral forelimb lameness in ponies. BMC Vet Res. 2018;14:151.

15. Manera ME, Carrillo JM, Batista M, Rubio M, Sopena J, Santana A, Vilar JM. Static Posturography: a new perspective in the assessment of lameness in a canine model. PLoS One. 2017;12(1):e0170692.

16. Temwichitr J, Leegwater PA, Hazewinkel HA. Fragmented coronoid process in the dog: a heritable disease. Vet J. 2010;185:123-9.

17. Cook CR, Cook JL. Diagnostic imaging of canine elbow dysplasia: a review. Vet Surg. 2009;38:144-53.

18. Scott H, Witte P. Investigation of lameness in dogs 1. Forelimb. Practice. 2011;33:20-7

19. Villamonte-Chevalier v BH, Broeckx B, Dingemanse W, Soler M, Van Ryssen $B$, Gielen I. Assessment of medial coronoid disease in 180 canine lame elbow joints: a sensitivity and specificity comparison of radiographic, computed tomographic and arthroscopic findings. BMC Vet Res. 2015;11:243.

20. Carrillo JM, Manera ME, Rubio M, Sopena J, Santana A, Vilar JM. Posturography and dynamic pedobarography in lame dogs with elbow dysplasia and cranial cruciate ligament rupture. BMC Vet Res. 2018;14:108. https://doi.org/10.1186/s12917-018-1435-y.

21. Asseman F, Caron O, Cremieux J. Is there a transfer of postural ability from specific to unspecific postures in elite gymnasts? Neurosci Lett. 2004;358:83-6.

22. Kernozek TW, LaMott EE, Dancisak MJ. Reliability of an in-shoe pressure measurement system during treadmill walking. Foot Ankle Int. 1996:17:204-9.

23. Galindo-Zamora V, Dziallas P, Wolf DC, Kramer S, Abdelhadi J, Lucas K, Nolte 1. Wefstaedt P. Evaluation of thoracic limb loads, elbow movement, and morphology in dogs before and after arthroscopic management of unilateral medial coronoid process disease. Vet Surg. 2014:43:819-28.

24. Basher A. Foot injuries in dogs and cats. Compend Cont Edu Pract vet $1 \mathrm{~b}$; 1994. p. 1159-76

25. Swaim SF. Management and bandaging of soft tissue injuries of dog and cat feet. J Am Anim Hosp Assoc. 1985;21:329-40.

26. Marghitu DB, Swaim SF, Rumph PF, Cojonaru D, Gillette RL, Scardino MS. Dynamics Analysis of Ground Contact Pressure of English Pointer Dogs. Nonlinear Dyn. 2003:33:253-65.

27. Johnston C, Back W. Hoof ground interaction: when biomechanical stimuli challenge the tissues of the distal limb. Equine Vet J. 2006:38:634-41.

28. Han TR, Paik NJ, Im MS. Quantification of the path of center of pressure (COP) using an F-scan in-shoe transducer. Gait Posture. 1999:10:248-54.

29. Molsa SH, Hyytiainen HK, Hielm-Bjorkman AK, Laitinen-Vapaavuori OM. Long-term functional outcome after surgical repair of cranial cruciate ligament disease in dogs. BMC Vet Res. 2014;10:266.

30. Oosterlinck M, Pille F, Back W, Dewulf J, Gasthuys F. A pressure plate study on fore and hindlimb loading and the association with hoof contact area in sound ponies at the walk and trot. Vet J. 2011;190:71-6.

Ready to submit your research? Choose BMC and benefit from:

- fast, convenient online submission

- thorough peer review by experienced researchers in your field

- rapid publication on acceptance

- support for research data, including large and complex data types

- gold Open Access which fosters wider collaboration and increased citations

- maximum visibility for your research: over $100 \mathrm{M}$ website views per year

At BMC, research is always in progress.

Learn more biomedcentral.com/submissions 